\title{
Prevalence of anopheline species and their Plasmodium infection status in epidemic-prone border areas of Bangladesh
}

\author{
Mohammad Shafiul Alam*, Md Gulam Musawwir Khan', Nurunnabi Chaudhury², Sharmina Deloer ${ }^{1}$, Forida Nazib', \\ A Mannan Bangali ${ }^{3}$, Rashidul Haque ${ }^{1}$
}

\begin{abstract}
Background: Information related to malaria vectors is very limited in Bangladesh. In the changing environment and various Anopheles species may be incriminated and play role in the transmission cycle. This study was designed with an intention to identify anopheline species and possible malaria vectors in the border belt areas, where the malaria is endemic in Bangladesh.
\end{abstract}

Methods: Anopheles mosquitoes were collected from three border belt areas (Lengura, Deorgachh and Matiranga) during the peak malaria transmission season (May to August). Three different methods were used: human landing catches, resting collecting by mouth aspirator and CDC light traps. Enzyme-linked immunosorbent assay (ELISA) was done to detect Plasmodium falciparum, Plasmodium vivax-210 and Plasmodium vivax-247 circumsporozoite proteins (CSP) from the collected female species.

Results: A total of 634 female Anopheles mosquitoes belonging to 17 species were collected. Anopheles vagus (was the dominant species (18.6\%) followed by Anopheles nigerrimus (14.5\%) and Anopheles philippinensis (11.0\%). Infection rate was found 2.6\% within 622 mosquitoes tested with CSP-ELISA. Eight (1.3\%) mosquitoes belonging to five species were positive for $P$. falciparum, seven (1.1\%) mosquitoes belonging to five species were positive for $P$. vivax -210 and a single mosquito (0.2\%) identified as Anopheles maculatus was positive for $P$. vivax-247. No mixed infection was found. Highest infection rate was found in Anopheles karwari (22.2\%) followed by An. maculatus (14.3\%) and Anopheles barbirostris (9.5\%). Other positive species were An. nigerrimus (4.4\%), An. vagus (4.3\%), Anopheles subpictus (1.5\%) and An. philippinensis (1.4\%). Anopheles vagus and An. philippinensis were previously incriminated as malaria vector in Bangladesh. In contrast, An. karwari, An. maculatus, An. barbirostris, An. nigerrimus and An. subpictus had never previously been incriminated in Bangladesh.

Conclusion: Findings of this study suggested that in absence of major malaria vectors there is a possibility that other Anopheles species may have been playing role in malaria transmission in Bangladesh. Therefore, further studies are required with the positive mosquito species found in this study to investigate their possible role in malaria transmission in Bangladesh.

\section{Background}

Throughout the world, there was an estimated 247 million malaria cases among 3.3 billion people at risk in 2006, causing nearly a million deaths, mostly of children under five years of age. In 2008, 109 countries were reported to be endemic for malaria. Bangladesh had an

\footnotetext{
* Correspondence: shafiul@icddrb.org

${ }^{1}$ Parasitology Laboratory, International Centre for Diarrhoeal Disease Research, Bangladesh (ICDDR,B), Dhaka-1212, Bangladesh
}

estimated 2.9 million malaria cases and 15,000 deaths in 2006. Although $72 \%$ of the population are at some risk of malaria, the risk is greatest in the east and north-east of the country in areas bordering India and Myanmar. The majority of suspected cases are unconfirmed; among those that are identified as malaria, more than $70 \%$ are Plasmodium falciparum [1]. Malaria is a major health burden in this remote, mountainous south-eastern region of Bangladesh. Malaria transmission in 
Bangladesh is mostly seasonal and concentrated in the border regions with India and Myanmar. Out of the total 64 administrative districts, 13 are located along the border areas with India and Myanmar where about 98\% of the total malaria morbidity and mortality reported from Bangladesh each year originate from these districts. [2]. According to passive data collected by Directorate General of Health Services (DGHS) of Bangladesh last ten years (1999-2008), the country's malaria situation remains almost steady with an annual incidence of $4 \%$ in the endemic districts.

In a recent survey, it has been found that among these 13 malaria endemic districts, the overall malaria prevalence rate was $3.1 \%$ based on Rapid Diagnostic Test (RDT). The prevalence of P. falciparum was $2.73 \%$ and the Plasmodium vivax $0.16 \%$ and mixed infection with $P$. falciparum and P. vivax was $0.19 \%$. The proportion of $P$. falciparum was $88.6 \%$, while $P$. vivax and mixed infection with these two species were 5.2 and $6.25 \%$, respectively. The overall malaria prevalence in Chittagong Hill Tracts was $11 \%$ [2].

The forests of Bangladesh have remained an area of intense malaria transmission providing a focus for re infection for the plains. Bangladesh has 34 species of anopheline mosquitoes[3]. Until 2009, only seven of these species were documented to be competent malaria vectors. Among these, four have been considered as the principal malaria vectors i.e. Anopheles baimaii (= Anopheles dirus D), Anopheles philippinensis, Anopheles sundaicus and Anopheles minimus s.l. [4]. Other species, such as Anopheles aconitus, Anopheles annularis and Anopheles vagus, were found to be capable of transmitting malaria during outbreak situations [5-7]. Although An. annularis and An. vagus are considered to be zoophilic, exophilic and exophagic in nature, they have been considered to maintain malaria transmission in the plain land. These two species were incriminated during epidemic situation in the flood plain areas of Bangladesh. Possibly they were incriminated due to low density of mammalian host except human [6,7].

DDT was banned in Bangladesh since 1985 and the number of malaria cases began to increase. Since then due to lack of adequate funds and programs, no control efforts maintained in the malaria endemic areas of Bangladesh[2]. Due to similar reason Malaria and Parasitic Disease Control Unit (M\&PDC) of DGHS could not carry out regular entomological investigation in the endemic areas. However, they carried out sporadic entomological surveillance, but did not have the opportunity to work on incrimination of other anopheline species. Therefore, this study was designed to obtain some information regarding prevalent anopheline species and possible malaria vectors in the border belt areas where the malaria situation is endemic in Bangladesh, the results of which are presented here.

\section{Methods \\ Study areas}

This study was conducted at three different border belt areas of Bangladesh with variable endemicity. These are Lengura (sub-district Kalmakanda: $25^{\circ} 46^{\prime} 0^{\prime \prime} \mathrm{N}, 90^{\circ} 54^{\prime}$ 0 " E) of Netrokona district, Deorgachh (including Chaklapunji tea estate; sub-district Chunarughat: $24^{\circ} 11^{\prime} 60^{\prime \prime}$ $\mathrm{N}, 91^{\circ} 31^{\prime} 0^{\prime \prime} \mathrm{E}$ ) of Habiganj district and Matiranga (subdistrict Matiranga: $23^{\circ} 7^{\prime} 19^{\prime \prime} \mathrm{N}, 91^{\circ} 52^{\prime} 36^{\prime \prime} \mathrm{E}$ ) of Khagrachhari district (Figure 1). Study areas were selected purposively based on the sites where DGHS conducted entomological investigation in recent past. Ecologically, Matiranga has predominately mixed thicket and dense forest and mixed evergreen and deciduous forest. Only $23 \%$ is cultivable land or fallow. Chunarughat has land cover with $51 \%$ tea plantations and $45 \%$ forest thickets interspersed with plantations like pineapple. Kalmakanda has land cover with very little forest (less than $0.1 \%$ ) and is primarily agricultural. More than $77 \%$ of the land is cultivable or fallow (source: Bangladesh Bureau of Statistics).

\section{Collection of Anopheles mosquitoes}

Anopheles mosquitoes were collected from (18.00-24.00 hours) both indoors and outdoors by human landing catches methods with the help of mouth aspirators[7]. Mosquitoes were collected from four houses per night on each of five successive nights once within the peak malaria transmission season (May to June). Four volunteers collected mosquitoes at each house two indoors and two outdoors. Every night houses was shifted randomly. Thus, in each study area for entomological surveillance, human landing catches (HLC) and resting collection were conducted in 20 households. Technical support for the entomological survey was provided by the M\&PDC of DGHS.

After completion of HLC and resting collection, CDC miniature light trap model 512 (origin: Jhon W. Hock Inc, USA) was also used for entomological investigation. Each trap was installed for at least 12 hours $(6 \mathrm{pm}$ to 6 am). Each night four trapping was conducted for five days of a week for each of the areas alternatively once in the peak season (May to October).

\section{Ethical consideration}

A written consent was obtained from the houses where entomological collections were made. For HLC, trained entomological technician working at DGHS were recruited. The mosquito collectors were monitored up to three months with a provision for treatment in case they got malaria, but such a case did not occur during 


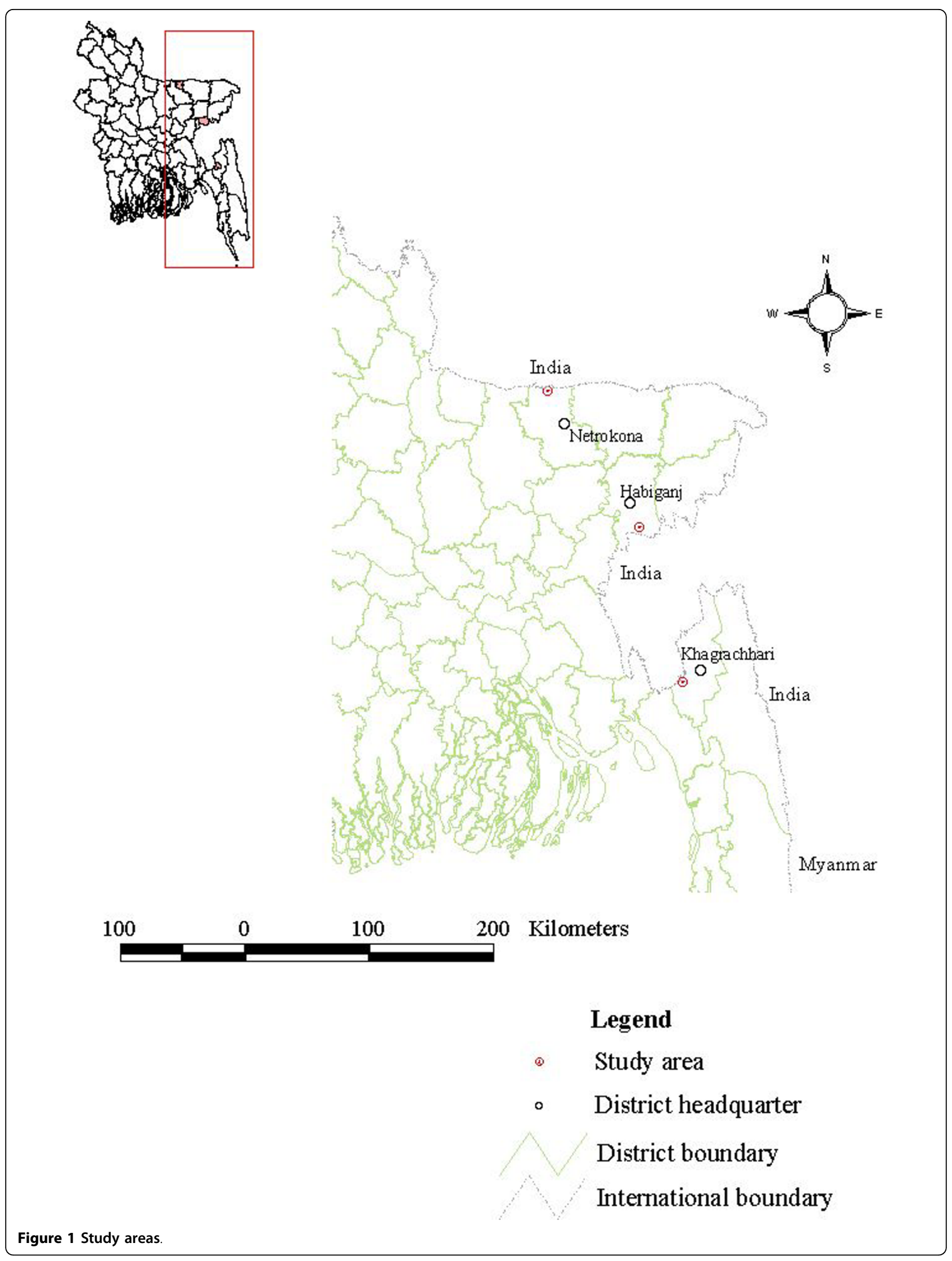


the reported period. Ethical approvals were obtained from regional WHO research review committee and ICDDR,B ethical review committee.

\section{Mosquito sample preparation}

The following morning after a catch, mosquitoes were sorted and identified. After identifying the species each mosquito was preserved in cryo-vial in silica gel in order to prevent microbial growth that can result in high background values.

\section{CSP ELISA}

Circumsporozoite protein (CSP) was detected using an enzyme-linked immunosorbent assay (ELISA), as described previously $[8,9]$. ELISAs were used to detect P. falciparum, $P$. vivax-210 (VK 210), and P. vivax-247 (VK 247) CSP in field caught mosquitoes. Plasmodium vivax has two distinct polymorphs in its CSP, VK210 and VK247, that are widespread in Southeast Asia and South America[10]. In areas where the two polymorphs coexist, intrinsic biological differences between the polymorphs may affect their survival. The ratios of VK210 to VK247 were significantly higher at the end of the non-transmission season than during the annual monsoon[11]. It was also reported that fluctuations in the proportion of mosquitoes infected with the two polymorphs may reflect humoral immune pressure on the VK247 strain [12]. In each test, positive control for each Plasmodium species were used and for negative control field caught male Anopheles mosquitoes were used. Monoclonal antibody (MAB) was obtained from the
Centers for Disease Control and Prevention (CDC), which were produced by Kirkegaard and Perry Laboratories (Atlanta, GA). Same batches of capture monoclonal antibodies were used in all tests. The absorbance of solution at $410 \mathrm{~nm}$ was determined $60 \mathrm{~min}$ after adding the substrate to Biorad ELISA plate reader. The cut-off was calculated by multiplying twice with the mean value of negative controls in respective tests. For ELISA positive mosquitoes tests were repeated to confirm it a positive.

\section{Results}

A total of 634 female anopheline mosquitoes belonging to 17 species were collected by different methods (Table 1). 403 mosquitoes were collected by CDC light trap and 231 mosquitoes by other methods (HLC and resting). Majority of the mosquitoes were collected by other methods were found resting in the cattle shed, indoor or outdoor of human dwellings. Anopheles vagus was the dominant species (18.6\%) followed by An. nigerrimus (14.5\%) and An. philippinensis (11.0\%). Matiranga represented with the highest number of species (15) and mosquitoes (511). Although Deorgachh and Lengura has simialr number of species (8), but mosquito numebrs were higher in Lengura (73) than Deorgachh (41). Since the numbers of mosquitoes in HLC were few, calculation for human biting rate was not performed.

CSP-ELISA was performed with 622 anopheline mosquitoes (remaining mosquitoes were kept as voucher specimen). 16 mosquitoes were positive in CSP-ELISA (Table 2). Thus, overall infection rate became $2.6 \%$

Table 1 List of anopheline species collected from three study areas by different methods

\begin{tabular}{|c|c|c|c|c|c|c|c|c|c|c|c|}
\hline \multirow[t]{2}{*}{ Species } & \multicolumn{3}{|c|}{ Matiranga } & \multicolumn{3}{|c|}{ Deorgachh } & \multicolumn{2}{|c|}{ Lengura** $^{* *}$} & \multicolumn{3}{|c|}{ Total } \\
\hline & LT & Others* & Total & LT & Others* & Total & LT & Total & LT & Others* & Total (\%) \\
\hline An. aconitus & 1 & 2 & 3 & 1 & 0 & 1 & 0 & 0 & 2 & 2 & $4(0.6)$ \\
\hline An. anularis & 0 & 0 & 0 & 0 & 0 & 0 & 1 & 1 & 1 & 0 & $1(0.2)$ \\
\hline An. barbirostris & 14 & 5 & 19 & 3 & 0 & 3 & 0 & 0 & 17 & 5 & $22(3.5)$ \\
\hline An. jamesii & 35 & 0 & 35 & 0 & 0 & 0 & 6 & 6 & 41 & 0 & $41(6.5)$ \\
\hline An. jeypurensis & 27 & 0 & 27 & 0 & 0 & 0 & 0 & 0 & 27 & 0 & $27(4.3)$ \\
\hline An. karwari & 0 & 0 & 0 & 0 & 0 & 0 & 10 & 10 & 10 & 0 & $10(1.6)$ \\
\hline An. kochi & 35 & 0 & 35 & 0 & 0 & 0 & 0 & 0 & 35 & 0 & $35(5.5)$ \\
\hline An. maculatus & 8 & 0 & 8 & 0 & 0 & 0 & 0 & 0 & 8 & 0 & $8(1.3)$ \\
\hline An. minimus s.l. & 1 & 0 & 1 & 0 & 0 & 0 & 0 & 0 & 1 & 0 & $1(0.2)$ \\
\hline An. niggerimus & 2 & 80 & 82 & 1 & 1 & 2 & 8 & 8 & 11 & 81 & $92(14.5)$ \\
\hline An. philippinensis & 54 & 0 & 54 & 6 & 3 & 9 & 7 & 7 & 67 & 3 & $70(11.0)$ \\
\hline An. subpictus & 1 & 58 & 59 & 0 & 6 & 6 & 1 & 1 & 2 & 64 & $66(10.4)$ \\
\hline An. tessellatus & 10 & 3 & 13 & 0 & 0 & 0 & 0 & 0 & 10 & 3 & $13(2.1)$ \\
\hline An. umbrosus & 56 & 1 & 57 & 5 & 0 & 5 & 0 & 0 & 61 & 1 & $62(9.8)$ \\
\hline An. vagus & 10 & 60 & 70 & 5 & 7 & 12 & 36 & 36 & 51 & 67 & 118 (18.6) \\
\hline An. varuna & 42 & 2 & 44 & 0 & 0 & 0 & 0 & 0 & 42 & 2 & $44(6.9)$ \\
\hline An. willmori & 13 & 0 & 13 & 0 & 3 & 3 & 4 & 4 & 17 & 3 & $20(3.2)$ \\
\hline $\mathrm{N}$ & 309 & 211 & 520 & 21 & 20 & 41 & 73 & 73 & 403 & 231 & $634(100)$ \\
\hline
\end{tabular}


Table 2 CSP-ELISA positive mosquitoes Infection rate according to species

\begin{tabular}{llllll}
\hline Species & No & Pf & Pv-210 & Pv-247 & Total \\
\hline An. aconitus & 3 & 0 & 0 & 0 & 0 \\
An. anularis & 1 & 0 & 0 & 0 & 0 \\
An. barbirostris & 21 & $1(4.8)$ & $1(4.8)$ & 0 & $2(9.5)$ \\
An. jamesii & 41 & 0 & 0 & 0 & 0 \\
An. jeypurensis & 27 & 0 & 0 & 0 & 0 \\
An. karwari & 9 & $1(11.1)$ & $1(11.1)$ & 0 & $2(22.22)$ \\
An. kochi & 35 & 0 & 0 & 0 & 0 \\
An. maculatus & 7 & 0 & 0 & $1(14.3)$ & $1(14.3)$ \\
An. minimus s.l. & 1 & 0 & 0 & 0 & 0 \\
An. niggerimus & 91 & $1(1.1)$ & $3(3.3)$ & 0 & $4(4.4)$ \\
An. & 69 & 0 & $1(1.4)$ & 0 & $1(1.4)$ \\
philippinensis & & & & & \\
An. subpictus & 65 & $1(1.5)$ & 0 & 0 & $1(1.5)$ \\
An. tessellatus & 12 & 0 & 0 & 0 & 0 \\
An. umbrosus & 61 & 0 & 0 & 0 & 0 \\
An. vagus & 116 & $4(3.4)$ & $1(0.9)$ & 0 & $5(4.3)$ \\
An. varuna & 44 & 0 & 0 & 0 & 0 \\
An. willmori & 19 & 0 & 0 & 0 & 0 \\
N & 622 & $8(1.28)$ & $7(1.1)$ & $1(0.2)$ & $16(2.6)$ \\
\hline
\end{tabular}

* include HLC and resting collection, ${ }^{* *} \mathrm{HLC}$ was not done

(16/622). Eight (1.3\%) mosquitoes belonging to five species were positive for P. falciparum, seven (1.1\%) mosquitoes belonging to five species were positive for VK210 and a single mosquito belonging to An. maculatus species was positive for VK 247. No mixed infection was found in this study. P. falciparum-positive anopheles species included one An. barbirostris, one An. karwari, four An. vagus, one An. nigerrimus, and one An. subpictus. VK 210 positive species included one An. barbirostris, one An. karwari, one An. vagus, three An. nigerrimus, and one An. philippinensis. According to species, the highest infection rate (Table 2) was observed in An. karwari $(2 / 9,22.2 \%)$ followed by An. maculatus (14.3\%), An. barbirostris (9.5\%), An. nigerrimus (4.4\%), An. vagus (4.3\%), An. subpictus (1.5\%) and An. philippinensis (1.4\%).

According to place from Matiranga 11 CSP-positive (2.2\%) mosquitoes had been identified in six species including An. barbirostris, An. subpictus, An. vagus, An. nigerrimus, An. maculatus and An. philippinensis (Table $3)$. In Lengura, five mosquitoes were identified CSP positive (6.9\%) belonging to two species including $A n$. karwari and An. vagus. In Deorgachh no mosquitoes were found CSP positive. Among 16 positive mosquitoes11 had blood on their abdomen, while seven had no visible blood meal (Table 4).

\section{Discussion}

Malaria transmission pattern in Bangladesh is still poorly understood. The on-going Malaria Control Programme in Bangladesh, stresses the fact of up-to-date information on malaria vectors. As a result the current vector control programmes are being implemented on little reliable report involved in malaria transmission. Successful implementation of a vector control programme in Bangladesh, the prevalence of infection with malaria sporozoites among the local anopheline mosquitoes is important, which will help to pinpoint the main vectors and other new vectors and to develop knowledge on the bionomics of the species involved in the disease transmission.

Anopheline mosquitoes were collected from the three study sites representing three geographically different endemic regions in Bangladesh. Anopheles vagus and An. philippinensis were previously incriminated as malaria vector in Bangladesh. There was, however, no previous report in favour of infections in An. karwari, An. maculatus, An. barbirostris, An. nigerrimus and An. subpictus in Bangladesh.

This study was conducted within a short period of time and mosquitoes were not collected on a seasonal basis. Although it was planned to collect by similar number of trapping in all three areas but failed to do so in Deorgachh. Thus, there might be a chance to miss some of existing anopheline species there. In Lengura, the highest prevalence rate (6.9\%) of CSP in Anopheles mosquitoes was found whereas in Matiranga the CSP prevalence rate was found $2.2 \%$. No sporozoite-positive mosquito was found in Deorgachh. In Derogachh, most traps were set up or conducted HLC in Chaklapunji tea garden, a famous entomological site where bionomics of $\mathrm{An}$. baimaii was studied in 70s $[13,14]$ where 15 anopheline species were recorded [14]. Anopheles baimaii, the major vector in tea garden area was not found in this investigation. Also the numbers of anopheline species were few in the tea garden area. Three reasons could be contributing such as effects of organic pesticide (deltamethrin) for the controlling of tea plant pests, deforestation and a delay in monsoon rains in Bangladesh in 2009. Due to delay in monsoon rain and prolonged dry season natural breeding places of An. baimaii and other anopheline species might have disappeared [15].

The presence of CSP in some anopheline species has been reported for the first time in Bangladesh, which is an imperative finding of this study. A total of seven species was found CSP-positive in the present study. The result of this study was compared with a recent study conducted in Assam state of north-eastern India, where there was evidence of CSP infection in An. karwari, An. maculatus, An. nigerrimus, An. barbirostris and An. subpictus [16].

Although An. barbirostris and An. subpictus were found positive in CSP ELISA in Sri Lanka [17], they had never been incriminated as malaria vector in Bangladesh. Anopheles vagus was highest in this study collection and also in CSP infection (5/116, 4.3\%): this species 
Table 3 Area wise CSP-ELISA positive rate

\begin{tabular}{|c|c|c|c|c|c|c|c|c|c|c|c|c|c|}
\hline \multirow{3}{*}{$\frac{\text { Species }}{\text { An. aconitus }}$} & \multicolumn{5}{|c|}{ Matiranga } & \multicolumn{4}{|c|}{ Deorgachh } & \multicolumn{4}{|c|}{ Lengura* } \\
\hline & LT & Others & Total & Positive & Pre (\%) & LT & Others & Total & Positive & Pre (\%) & LT & Positiv & Pre (\%) \\
\hline & 1 & 1 & 2 & 0 & 0 & 1 & 0 & 1 & 0 & 0 & 0 & 0 & - \\
\hline An. anularis & 0 & 0 & 0 & - & - & 0 & 0 & 0 & - & - & 1 & 0 & 0 \\
\hline An. barbirostris & 13 & 5 & 18 & 2 & 11.1 & 3 & 0 & 3 & 0 & 0 & 0 & - & - \\
\hline An. jamesii & 35 & 0 & 35 & 0 & 0 & 0 & 0 & 0 & - & - & 6 & 0 & 0 \\
\hline An. jeypurensis & 27 & 0 & 27 & 0 & 0 & 0 & 0 & 0 & - & - & 0 & - & - \\
\hline An. karwari & 0 & 0 & 0 & - & - & 0 & 0 & 0 & - & - & 9 & 2 & 22.2 \\
\hline An. kochi & 35 & 0 & 35 & 0 & 0 & 0 & 0 & 0 & - & - & 0 & - & - \\
\hline An. maculatus & 7 & 0 & 7 & 1 & 14.3 & 0 & 0 & 0 & - & - & 0 & - & - \\
\hline An. minimus s.l. & 1 & 0 & 1 & 0 & 0.0 & 0 & 0 & 0 & - & - & 0 & - & - \\
\hline An. niggerimus & 2 & 79 & 81 & 4 & 0.0 & 1 & 1 & 2 & 0 & 0 & 8 & 0 & 0 \\
\hline An. philippinensis & 54 & 0 & 54 & 1 & 1.9 & 5 & 3 & 8 & 0 & 0 & 7 & 0 & 0 \\
\hline An. subpictus & 0 & 58 & 58 & 1 & 1.7 & 0 & 6 & 6 & - & - & 1 & 0 & 0 \\
\hline An. tessellatus & 9 & 3 & 12 & 0 & 0 & 0 & 0 & 0 & - & - & 0 & - & - \\
\hline An. umbrosus & 55 & 1 & 56 & 0 & 0 & 5 & 0 & 5 & 0 & 0 & 0 & - & - \\
\hline An. vagus & 9 & 59 & 68 & 2 & 2.9 & 5 & 7 & 12 & 0 & 0 & 36 & 3 & 8.3 \\
\hline An. varuna & 42 & 2 & 44 & 0 & 0 & 0 & 0 & 0 & - & - & 0 & - & - \\
\hline An. willmori & 13 & & 13 & 0 & 0 & 0 & 2 & 2 & - & - & 4 & 0 & 0 \\
\hline $\bar{N}$ & 303 & 208 & 511 & 11 & 2.2 & 20 & 19 & 39 & 0 & 0 & 72 & 5 & 6.9 \\
\hline
\end{tabular}

LT: Light trap, Others: HLC and resting collection

* HLC was not done

has been incriminated as malaria vector in Bangladesh [7]. Although An. aconitus, An. annularis, An. jeyporiensis and $A n$. varuna also appeared to have vector potential $[5,6,18]$, but CSP was not detected in these species in the present study.

CSP-ELISA has emerged as a useful tool for vector detection, indicating that several species once considered un-important in the epidemiology of malaria, such as $A n$. subpictus and An. vagus in Sri Lanka [17]. In this study,
An. nigerrimus was found CSP-positive, which is probably a first-time report in this region, while the species remains the principal malaria vector in the Indo-Chinese Hills and the Malaysian Zones(Varma MG: Geographical distribution of arthropod borne disease and their principal vectors, unpublished document WHO/VBC/89967). Similarly, $A n$. karwari is considered a secondary vector in the Australian region [19], but its vectorial status in South-East Asia was unknown. There is still remaining controversy for

Table 4 Summary table for positive anopheles female mosquitoes in CSP-ELISA from border belt areas of Bangladesh

\begin{tabular}{|c|c|c|c|c|c|c|}
\hline Sample ID & Species name & Positive type & Fed* & Place of collection & Collection date & Collection type \\
\hline 38 & An. nigerrimus & Pv-210 & 0 & Matiranga & 14.05 .09 & Others \\
\hline 58 & An. nigerrimus & Pv-210 & 1 & Matiranga & 14.05 .09 & Others \\
\hline 67 & An. nigerrimus & $P \vee-210$ & 1 & Matiranga & 14.05 .09 & Others \\
\hline 70 & An. nigerrimus & Pf & 1 & Matiranga & 14.05 .09 & Others \\
\hline 134 & An. subpictus & Pf & 1 & Matiranga & 14.05 .09 & Others \\
\hline 185 & An. karwari & Pf & 0 & Lengura & 12.08 .09 & $\mathrm{LT}$ \\
\hline 187 & An. karwari & $P \vee-210$ & 1 & Lengura & 12.08 .09 & $\mathrm{LT}$ \\
\hline 201 & An. vagus & Pf & 1 & Lengura & 12.08 .09 & $\mathrm{LT}$ \\
\hline 209 & An. vagus & $P f$ & 0 & Lengura & 12.08 .09 & $\mathrm{LT}$ \\
\hline 228 & An. vagus & Pf & 1 & Lengura & 12.08 .09 & $\mathrm{LT}$ \\
\hline 264 & An. vagus & $P \vee-210$ & 1 & Matiranga & 14.05 .09 & Others \\
\hline 272 & An. vagus & Pf & 1 & Matiranga & 14.05 .09 & Others \\
\hline 314 & An. maculatus & PV-247 & 0 & Matiranga & 19.06 .09 & $\mathrm{LT}$ \\
\hline 406 & An. barbirostris & Pf & 1 & Matiranga & 19.06 .09 & $\mathrm{LT}$ \\
\hline 414 & An. barbirostris & $P \vee-210$ & 1 & Matiranga & 19.06 .09 & $\mathrm{LT}$ \\
\hline 438 & An. philippinensis & Pv-210 & 0 & Matiranga & 19.06 .09 & $\mathrm{LT}$ \\
\hline
\end{tabular}

* 0 = unfed; 1 = blood fed; LT = Light trap 
CSP-ELISA particularly due to its false positive results in previous studies. Thus, positivity in a CSP-ELISA should not be taken as the only criterion in confirming the vector status of an Anopheles species [20-22].

The present result does not report any infection evidence in An. minimus s.l. This might be due to only one mosquito of this species was tested. In a recent study conducted in Chakaria of Bangladesh, which is geographically similar to Matiranga, a higher percentage of An. minimus s.l. was caught $(97.3 \% ; 651 / 669)$, of which 19 were positive for Plasmodium infection by the microtiter plate hybridization (MPH) method[23]. Before 1950, An. minimus s.l. was the principal vector recognized in Bangladesh, but its population declined due to the routine spraying of DDT, to which it remains susceptible[14]. In recent past, the density of An. minimus s.l. was negligible, as observed in a few sporadic entomological investigations carried out by M\&PDC (personal communication with NP Maheswary, a veteran entomologist). Hence, the higher number of An. minimus s.l. reported in Chakaria might be due to misidentification. A similar situation occurred in Vietnam where formally identified An. minimus s.l. was found to be Anopheles varuna [24]. A reasonable number of An. varuna in the present study is also supportive to this fact.

\section{Conclusions}

Findings of this study suggested that anopheline species other than An. minimus s.l. and An. baimaii might have a role in the transmission of malaria in endemic areas of Bangladesh. The detection of CSP in some anopheline species should be taken into consideration for further studies to investigate their possible role in malaria transmission in Bangladesh.

\section{Acknowledgements \\ This investigation received financial assistance from WHO/SEARO in collaboration with UNICEF/UNDP/World Bank/WHO Special Programme for Research and Training in Tropical Diseases (TDR). \\ We are grateful to the staffs of M\&PDC for their active participation during entomological investigation. We are also grateful to Sumit Chakma, Milka Patracia Podder, AEM Rubayet Elahi and SM Khaled Abdullah for their contribution in this study. We would also like to acknowledge the contributions of Touhid Uddin Ahmed and Rajib Chowdhury for critically reviewing this manuscript.}

\section{Author details \\ 'Parasitology Laboratory, International Centre for Diarrhoeal Disease Research, Bangladesh (ICDDR,B), Dhaka-1212, Bangladesh. ${ }^{2}$ Malaria and Parasitic Disease Control Unit, Directorate General of Health Services, Mohakhali, Dhaka-1212, Bangladesh. ${ }^{3}$ World Health Organization, Dhaka, Bangladesh.}

\section{Authors' contributions}

MSA conceptualized and designed the study collected and identified sample, analyzed data, drafted the manuscript and made final revisions. MGMK, SD, FN did sample analysis and made critical revision of the manuscript. NC organized the field activities, analysed data and helped in the revision of the manuscript. AMB and RH participated in study design, critical analysis of data and helped in drafting the manuscript. All authors read the final manuscript and approved.

\section{Competing interests}

The authors declare that they have no competing interests.

Received: 19 November 2009

Accepted: 14 January 2010 Published: 14 January 2010

\section{References}

1. Aregawi M, Cibulskis R, Otten M: World Malaria Report 2008. World Health Organization 2008

2. Haque U, Ahmed SM, Hossain S, Huda M, Hossain A, Alam MS, Mondal D, Khan WA, Khalequzzaman M, Haque R: Malaria prevalence in endemic districts of Bangladesh. PLoS One 2009, 4:e6737.

3. Ahmed TU: Checklist of the mosquitoes of Bangladesh. Mosquito Systematics 1987, 19:187-200.

4. Elias M, Dewan ZAR, Ahmed R: Vectors of malaria in Bangladesh. J Prev Social Med 1982, 1:20-28.

5. Maheswary NP, Habib MA, Elias M: Incrimination of Anopheles aconitus Donitz as a vector of epidemic malaria in Bangladesh. Southeast Asian $J$ Trop Med Public Health 1992, 23:798-801.

6. Maheswary NP, Khan Z, Molla FR, Haq MI: Incrimination of Anopheles annularis van der Wulp-1854 as an epidemic malaria vector in Bangladesh. Southeast Asian J Trop Med Public Health 1993, 24:776-778.

7. Maheswary NP, Majumdar S, Chowdhury AR, Faruque MS, Montanari RM: Incrimination of Anopheles vagus Donitz, 1902 as an epidemic malaria vector in Bangladesh. Indian J Malariol 1994, 31:35-38.

8. Wirtz RA, Burkot TR, Graves PM, Andre RG: Field evaluation of enzymelinked immunosorbent assays for Plasmodium falciparum and Plasmodium vivax sporozoites in mosquitoes (Diptera: Culicidae) from Papua New Guinea. J Med Entomol 1987, 24:433-437.

9. Wirtz RA, Zavala F, Charoenvit Y, Campbell GH, Burkot TR, Schneider I, Esser KM, Beaudoin RL, Andre RG: Comparative testing of monoclonal antibodies against Plasmodium falciparum sporozoites for ELISA development. Bull World Health Organ 1987, 65:39-45.

10. Rosenberg R, Wirtz RA, Lanar DE, Sattabongkot J, Hall T, Waters AP, Prasittisuk C: Circumsporozoite protein heterogeneity in the human malaria parasite Plasmodium vivax. Science 1989, 245:973-976.

11. Suwanabun N, Sattabongkot J, Wirtz RA, Rosenberg R: The epidemiology of Plasmodium vivax circumsporozoite protein polymorphs in Thailand. Am J Trop Med Hyg 1994, 50:460-464

12. Burkot TR, Wirtz RA, Paru R, Garner P, Alpers MP: The population dynamics in mosquitoes and humans of two Plasmodium vivax polymorphs distinguished by different circumsporozoite protein repeat regions. Am J Trop Med Hyg 1992, 47:778-786.

13. Rosenberg R: Forest malaria in Bangladesh. III. Breeding habits of Anopheles dirus. Am J Trop Med Hyg 1982, 31:192-201.

14. Rosenberg R, Maheswary NP: Forest malaria in Bangladesh. II. Transmission by Anopheles dirus. Am J Trop Med Hyg 1982, 31:183-191

15. Earth Observatory. http://earthobservatory.nasa.gov//OTD/view.php? id $=39481$.

16. Prakash A, Bhattacharyya DR, Mohapatra PK, Mahanta J: Role of the prevalent Anopheles species in the transmission of Plasmodium falciparum and P. vivax in Assam state, north-eastern India. Ann Trop Med Parasitol 2004, 98:559-568.

17. Amerasinghe PH, Amerasinghe FP, Konradsen F, Fonseka KT, Wirtz RA: Malaria vectors in a traditional dry zone village in Sri Lanka. Am J Trop Med Hyg 1999, 60:421-429.

18. Dash AP, Adak T, Raghavendra K, Singh OP: The biology and control of malaria vectors in India. Current Science 2007, 92:1571-1578.

19. Service MW: Mosquitoes (Culicidae). Medical insects and arachnids London: The Natural History MuseumLane R, Crosskey R 1993.

20. Beier JC, Perkins PV, Wirtz RA, Whitmire RE, Mugambi M, Hockmeyer WT: Field evaluation of an enzyme-linked immunosorbent assay (ELISA) for Plasmodium falciparum sporozoite detection in anopheline mosquitoes from Kenya. Am J Trop Med Hyg 1987, 36:459-468.

21. Lochouarn L, Fontenille D: ELISA detection of malaria sporozoites: falsepositive results in Anopheles gambiae s.l. associated with bovine bloodmeals. Trans R Soc Trop Med Hyg 1999, 93:101-102. 
22. Somboon P, Morakote N, Koottathep S, Trisanarom U: Detection of sporozoites of Plasmodium vivax and Plasmodium falciparum in mosquitoes by ELISA: false positivity associated with bovine and swine blood. Trans R Soc Trop Med Hyg 1993, 87:322-324.

23. Tangin A, Komichi Y, Wagatsuma Y, Rashidul H, Wataya Y, Kim HS: Detection of malaria parasites in mosquitoes from the malaria-endemic area of Chakaria, Bangladesh. Biol Pharm Bull 2008, 31:703-708.

24. Van Bortel W, Harbach RE, Trung HD, Roelants P, Backeljau T, Coosemans M: Confirmation of Anopheles varuna in Vietnam, previously misidentified and mistargeted as the malaria vector Anopheles minimus. Am J Trop Med Hyg 2001, 65:729-732.

doi:10.1186/1475-2875-9-15

Cite this article as: Alam et al:: Prevalence of anopheline species and their Plasmodium infection status in epidemic-prone border areas of Bangladesh. Malaria Journal 2010 9:15.

\section{Submit your next manuscript to BioMed Central} and take full advantage of:

- Convenient online submission

- Thorough peer review

- No space constraints or color figure charges

- Immediate publication on acceptance

- Inclusion in PubMed, CAS, Scopus and Google Scholar

- Research which is freely available for redistribution

Submit your manuscript at www.biomedcentral.com/submit 